\title{
The mapping of smartphone usage by adolescent with visual impairment
}

\author{
Hanny Hafiar ${ }^{1}$, Priyo Subekti ${ }^{2}$, Aat Ruchiat Nugraha ${ }^{3}$ \\ \{hanny.hafiar@unpad.ac.id $\left.{ }^{1}\right\}$ \\ 1,2,3Universitas Padjadjaran, Bandung, Indonesia
}

\begin{abstract}
As millennial generations, adolescents with visually impaired also have attachments to smartphones. Limited vision, does not make them lagging in technology. As evidence, most teenagers in the complex of Wiyataguna Bandung which is the rehabilitation centers for the people awith visually impaired, they use the smartphone not only for communication but also for other needs. Therefore, this study aims to determine the use of smartphones among adolescents with visually impaired. The method used is the descriptive, distributing questionnaires and conducting interviews. The results obtained : the use of smartphones for calculator features; camera; location search; karaoke; download songs and videos; accessing novels, e-books, and other text; watching soap operas, streaming football; radio; online shop; photo; lesson info; time and date; game; take notes; telephone connection; TalkBack; record; sending messages; social media. While the use of applications on smartphones that are data plan or phone credit based includes google searching, social media, telephone, SMS, Radio streaming, online games, and YouTube. The perceived obstacles include technical/skill disruptions, products, applications, and networks. These results indicate the existence of smartphone technology capabilities by adolescents with visually impaired, but still need guidance, direction, and supervision so they can be smart in using media technology.
\end{abstract}

Keywords: Smartphone, Millennial, Visual Impairment

\section{Introduction}

The smartphone is one of the communication technologies that provide various other functions besides simply communicating via the telephone connection and sending SMS. Through the help of an internet connection, a smartphone can be used for various information receiving and sending needs. Smartphones can also be used as a device to produce, process, and store data in the form of text, sound, images, and impressions. With its multi-functional nature, smartphones have become one of the devices that have been taken advantage of by humans, ranging from children to the elderly.

Unlike children who do not have deep knowledge of smartphones, or parents who tend to lack expertise and ability to explore the latest technology, adolescents tend to be easier and faster to adapt to smartphone devices and their development. Including among adolescents who have disabilities such as adolescents with visually impaired. Although in its use, people with disabilities often need rehabilitation devices as additional tools. Cook and Hussey stated that the fundamental goal of rehabilitation tools is to increase the independence of disability 
[1]. Thus, it is expected that the existence of the results of the design in the form of rehabilitation devices found on smartphones can create disability independence in communicating and sharing information.

Technology design is based more on the needs of the general public, so that certain technological devices are often inappropriate and difficult to use for individuals with special needs, especially for those who still in the children's group category, because designing assistive technology for children with disabilities is quite complex due to the lack of knowledge about how they use technology [2], including their needs and expectations for technology as a tool that facilitates human activities during adolescence.

Therefore, the design and development of such devices should not only be based on user skills, but also about the objectives of the activity and the context or environment of its use [1], including smartphone users who are in their teens.

In principle, the internet not only has the potential to improve their ability to carry out daily tasks, but also to overcome the visual impairments experienced and feel a part of being socially integrated with the general community. However, the rapid advancement of technology is feared to be able to further expand the digital discrepancy if it is not accompanied by efforts to overcome the obstacles experienced by disabilities in using the internet [3]. Based on this, we need an understanding of the functions and uses of smartphones for adolescents with visually impaired along with the needs of features that can be utilized both those demanding the use of quota/phone credit and those that are not, and the obstacles they face. In addition to the availability of features that have accessibility for the visually impaired, there are other issues that need to be considered, namely expertise in smartphone use that needs to be taught by parents and teachers in schools, because the lack of teachers' ability to socialize technology and various applications of assistance can negatively affect students with visual impairments because it inhibits the development of assistive technology skills, which ultimately results in graduates and prospective unskilled workforce [4]. Therefore, parents and teachers of young people with disabilities need to understand the available tools, so that young people with disabilities can optimize the use of their smartphones. Based on these thoughts, this study aims to determine the features that are used and utilized by young people with disabilities, both those who demand the use of quota/phone credit and those who do not, and the obstacles they face.

\section{Method}

This research was conducted by disseminating a number of questionnaires filled out by respondents from being read out and accompanied when filling in. So when filling out the questionnaire the researcher can immediately ask questions and conduct interviews with respondents. The research was conducted at the Wiyataguna Bandung complex, which is a national rehabilitation center for blind people. Most of the residents of the community are students who are studying from PAUD (Early Childhood Education Program) to high school, and dormitory residents who are educated in college, or who are currently taking skills training as an effort to provide expertise that can be used after the rehabilitation phase. A total of 41 respondents filled out the questionnaire and gave answers and their reasons. Furthermore, data are classified and displayed in graphical form and analyzed to get a more comprehensive understanding.

\section{Results and discussion}


Adolescents as the millennials have certain characteristics that are different from the previous generation. One of the characteristics of this generation is their high ability to adapt to technology, including smartphones. This characteristic can also be seen in adolescents with visual impairment. Adolescents with visual impairments who are in the Wiyataguna Bandung complex look familiar and no stranger with the smartphones, including special features intended to facilitate the visually impaired in using this communication media, which is presented in the following picture:

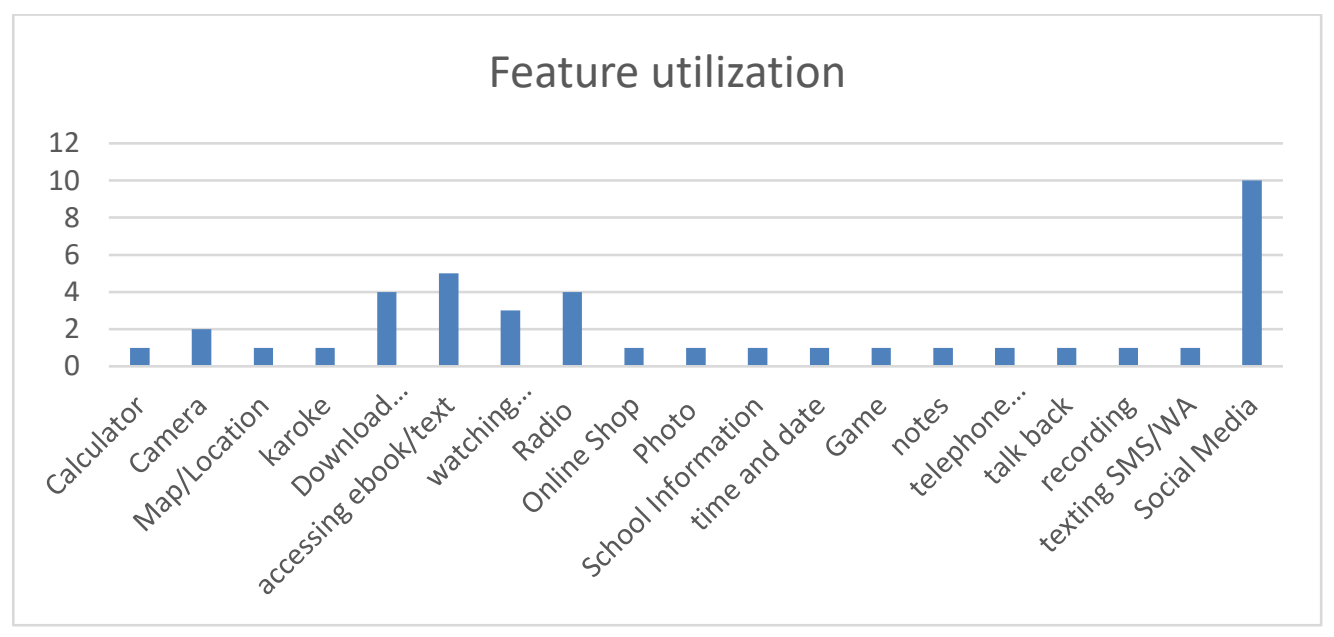

Fig. 1 Feature Utilization

Based on the results of the field data, it is known that there are several smartphone features mentioned by the respondents who are adolescents with visual impairments, including a calculator; camera; location search; karaoke; download both songs and videos; accessing novels, e-books, and other text stories; watching soap operas, streaming football; radio; online shop; photo; lesson info; time and date; game; take notes; telephone connection; talkback; record; sending message; social media.

Of the many features, social media features are the highest features mentioned by respondents, followed by features of accessing novels, and other text sources, and radio and downloading songs or videos. This shows that there is a considerable amount of social media use by adolescents with visual impairments. The tendency to use social media is expected to empower the potential and increase the social interaction of adolescents with their environment, both fellow visual impairments people and the general public. The social networking sites are considered to have the potential to empower people with disabilities [5].

On the use of social media, adolescents with visual impairments as the users feel the need to install a picture profile with a specific purpose. Therefore, there are times when young people with disabilities try to take pictures, both selfie and taking another image object. As stated in a previous research that, smartphone users are able to direct the camera in the desired direction and to produce the intended image [6], including adolescents with visual impairments who were respondents in this study which mentioned that their smartphones are often used to make photographs. Photo as a digital image is widely used for various purposes in contemporary society, including education, profession, business, participation, entertainment, and social interaction. But most digital images remain inaccessible to the 
visually impaired [7]. The problem, trying to be solved by various application developers. Now, there is the development of PictureSensation, which is a mobile application for exploring hapto-acoustic images designed for people who have visual impairments to get perceptual access directly to images through signals [8]. In addition, a photo-to-sound application for the blind has been developed or Camera Reading for people with visual impairments, the main goal of which is the development of mobile applications that allow blind users to "read" text (a piece of paper, signals, etc.) [9] In addition to social media and photos, the application also mentioned by adolescents with visual impairments which also provide convenience is the navigation application. Along with the increasing concern of application developers on the needs of people with visual impairments, there has been an innovative information and navigation system for smartphones to help direct people with visual impairments when entering new areas or environments (eg shopping centers, office buildings, and other public facilities) [10]. This means that in this digital era, many assistive devices have been created that make it easier for people with visual impairments to take advantage of smartphone functions as expressed by respondents in this study.

Social media is the most frequently accessed applications on a smartphone based on the explanation of the respondent, even though they have to buy data plan or phone credit. This means that the motivation level for accessing social media is quite high because they still try to access the application even if they have to pay a certain amount to purchase data plan and phone credit. There are studies that examine the motivations, challenges, interactions, and experiences of people with visual impairments related to visual content in Social Networking Services including social media, the results of which state that people with visual impairments continue to strive to overcome obstacles to accessing visual content in various ways and strategies. This shows that social media is something that is considered important, including by adolescents with visual impairments in this study.

There is other research that examines free usage instructions and applications in the Google Play application store [11], with the results stating that some applications, even with instructions for using assistive devices, sometimes require the help of others. This is in accordance with additional data obtained in research that there are times when installing new applications or operationalizing new features, adolescents with visual impairments ask for direction and assistance from others, both sighted people and other people with visual impairments who still have visions' left or who have better technological abilities. This means that smartphones with a variety of the latest supporting devices still have their own obstacles in the eyes of adolescents with visual impairments which can be described in the following picture: 


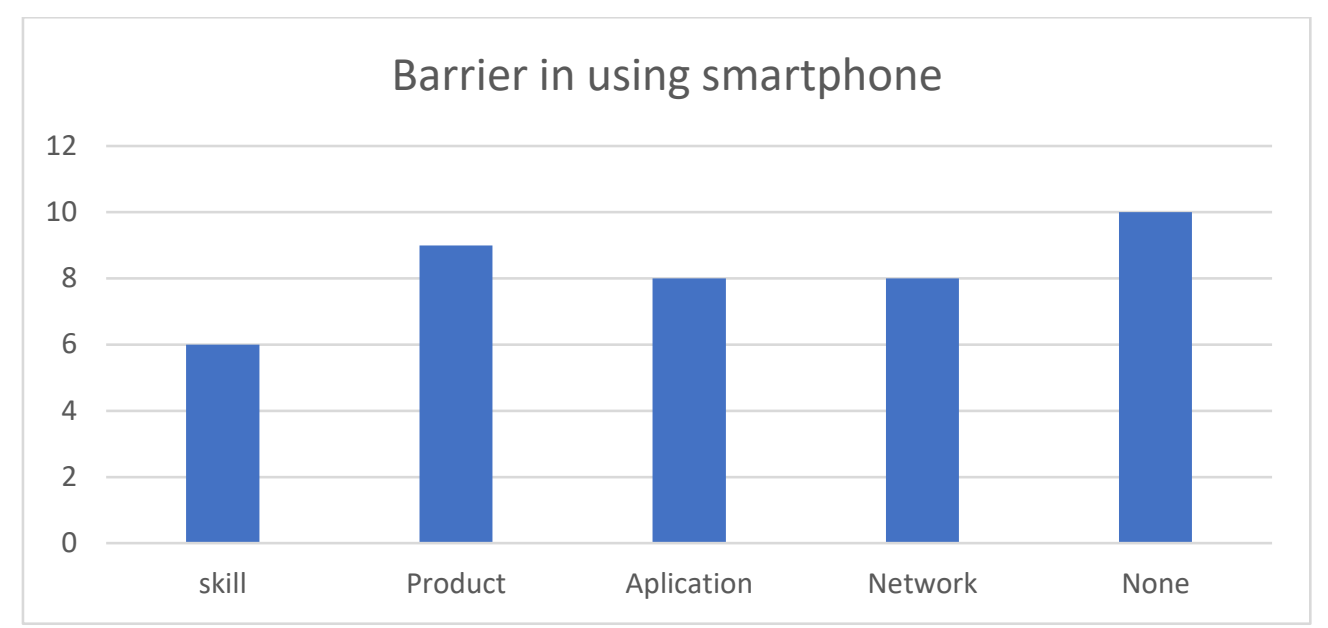

Fig. 2 Barrier in using smartphone

Based on field data collection, it is known that perceived obstacles include the disruption of technical/skill, product, application, and network. On barriers that are technical or skillrelated, it is stated by adolescents with visual impairments that many obstacles to smartphone use are caused by their low knowledge and expertise in optimizing smartphones, and the awkwardness of trying to explore all the features on their smartphones, including the video call feature. A study has been conducted that seeks to compare the level of acceptance, attitudes, and intensity of smartphone use among people with visual impairments groups who have and have not experienced using the video call feature mentioning that blind group that have and have the experience to use video calls via smartphones both have acceptance of the technology. The difference is the attitude and intensity of use [12].

This is in line with the findings of this study that in general, adolescents with visual impairments want to have the ability to utilize the functions of their smartphones optimized, but it is hampered by the standards of attitudes in most adolescents with visual impairments, namely in the form of concerns based on their experience that the exploration process actually creates new problems such as lost data, changes to settings, even the phone becomes hanged caused by a keypress error. Therefore, some teenagers choose to act and behave passively in exploring smartphone functions to minimize risk. Error keystrokes, related to the texting method on most touchscreen devices developed without taking into account the needs of people who have a low vision [13], especially the totally blind. So that the rapid development of aid applications in using technology, it turns out that some blind users still face several problems such as opening and using the touchscreen smartphone function [14]. This requires special thinking, considering globally, the number of people with visual impairments is quite large and the growth rate is quite high. So that it has an impact on the number of assistive technologies that are being and have been developed to help the people with visual impairments, but they still have difficulty accessing technology, because the development of these technologies tends to be more developed through the perspective of the general public [15]. Therefore, it's time for technology developers to try to review technology from the perspective of people with visual impairments and other disabilities as users who also have the same rights as the general public. 


\section{Conclusion}

The results obtained are related to the smartphone features which are used and mentioned first by the respondent when questions are asked, including the use of smartphones for calculator features; camera; location search; karaoke; download both songs and videos; accessing novels, e-books, and other text stories; watching soap operas, streaming football; radio; online shop; photo; lesson info; time and date; game; take notes; telephone connection; talkback; record; sending message; social media. While the use of applications on smartphones, which are used with a data plan or phone credit basis includes google searching, social media, telephone, SMS, Radio streaming, online games, and YouTube. The perceived obstacles include technical / skill disruptions, products, applications, and networks. Based on these results indicate the existence of smartphone technology capabilities by adolescents with visually impaired, but they still need the guidance, direction, and supervision so that adolescents with visually impaired can be smart in using media technology.

\section{References}

[1] A. M. Echenique et al., "Inclusive Educative Technologies, for people with disabilities," $J$. Phys. Conf. Ser., vol. 705, pp. 1-10, 2016.

[2] E. Brulé, G. Bailly, A. Brock, F. Valentin, G. Denis, and C. Jouffrais, "MapSense : MultiSensory Interactive Maps for Children Living with Visual Impairments," Proc. Annu. ACM Conf. Hum. Factors Comput. Syst., 2016.

[3] P. Okonji, M. Lhussier, C. Bailey, and M. Cattan, "Internet use: Perceptions and experiences of visually impaired older adults," J. Soc. Incl., vol. 6, no. 1, pp. 120-145, 2015.

[4] Y.-T. Siu and V. S. Morash, "Teachers of Students with Visual Impairments and Their Use of Assistive Technology: Measuring the Proficiency of Teachers and Their Identification with a Community of Practice," J. Vis. Impair. Blind., vol. 108, no. 5, pp. 384-398, 2014.

[5] C. Shpigelman and C. J. Gill, "Facebook Use by Persons with Disabilities," J. Comput. Mediat. Commun., vol. 19, pp. 610-624, 2014.

[6] S. Sahasrabudhe, R. Singh, and D. Heath, "Innovative Affordances for Blind Smartphone Users : A Qualitative Study,” J. Technol. Pers. with Disabil., pp. 145-156, 2016.

[7] M. R. Morris, J. Johnson, C. L. Bennett, and E. Cutrell, "Rich Representations of Visual Content for Screen Reader Users," Proc. 2018 CHI Conf. Hum. Factors Comput. Syst., 2018.

[8] M. Banf, R. Mikalay, B. Watzke, and V. Blanz, "PictureSensation - a mobile application to help the blind explore the visual world through touch and sound," J. Rehabil. Assist. Technol. Eng., vol. 3, pp. 1-10, 2016.

[9] R. Neto and N. Fonseca, "Camera Reading for Blind People," Procedia Technol., vol. 16, pp. 1200-1209, 2014.

[10] J. Cecílio, K. Duarte, and P. Furtado, "BlindeDroid: An information tracking system for realtime guiding of blind people," Procedia Comput. Sci., vol. 52, no. 1, pp. 113-120, 2015.

[11] M. Bülbül, N. Yiğit, and B. Garip, "Adapting smart phone applications about physics education to blind students," J. Phys. Conf. Ser., vol. 707, no. 1, 2016.

[12] T. Chanjaraspong, "Acceptance factors for the use of video call via smartphone by blind people," Kasetsart J. Soc. Sci., vol. 38, no. 1, pp. 81-87, 2017.

[13] M. Alnfiai and S. Sampalli, "BrailleEnter: A Touch Screen Braille Text Entry Method for the Blind," Procedia Comput. Sci., vol. 109, no. 2016, pp. 257-264, 2017.

[14] M. C. Rodriguez-Sanchez, M. A. Moreno-Alvarez, E. Martin, S. Borromeo, and J. A. Hernandez-Tamames, "Accessible smartphones for blind users: A case study for a wayfinding system," Expert Syst. Appl., vol. 41, no. 16, pp. 7210-7222, 2014.

[15] H. K. Kim, S. H. Han, J. Park, and J. Park, "The interaction experiences of visually impaired people with assistive technology: A case study of smartphones," Int. J. Ind. Ergon., vol. 55, pp. 
22-33, 2016. 